\title{
Commentary on 'Comparison of a low Hartmann's procedure with low colorectal anastomosis with and without defunctioning ileostomy after radiotherapy for rectal cancer: results from a national registry'
}

doi:10.1111/codi.13289

An indication for primary end-colostomy after neoadjuvant therapy for low rectal cancer can be due to the tumor (and an abdominoperineal amputation is performed), due to patients comorbidity and fear of complications after a very low anastomosis, or because function and quality of life are judged to be poor with a low anastomosis. In the Dutch population-based analysis in this issue [1] $27.8 \%$ of patients with a rectal cancer $\leq 10 \mathrm{~cm}$ having neoadjuvant therapy underwent a low Hartmann's procedure (LHP). The remaining patients had a low anastomosis with or without diverting ileostomy $(51 \%$ and $20.2 \%$, respectively). The authors demonstrated fewer abdominal infective complications and reoperations with LHP. A low anastomosis without diverting ileostomy was an independent risk factor of re-operation. Patients undergoing LHP had a higher $\mathrm{Rl}$ resection rate and were significantly older with more comorbidities resulting in a higher 30-day mortality. However, in multivariate analyses LHP was not an independent predictor of mortality.

One third of patients with a rectal cancer after neoadjuvant therapy and a potentially restorative low anterior resection (LAR) will have a primary end-colostomy in Holland (abdominal perineal resections excluded). This number seems very high. The risk of definitive stoma formation after low LAR or intersphincteric resection in very low cancers $(0-6 \mathrm{~cm}$ from the anal verge) was $11 \%$ at 1 year and $22 \%$ at 10 years in a recent study [2]. Incontinence (8\%) and anastomotic morbidity (6.5\%) were the main reasons for stoma formation. Both, age $>65$ years and surgical morbidity were independent risk factors. In other words, at 10 years $78 \%$ of patients with a low anastomosis do not have a stoma and presumably preserve adequate continence. In the Dutch study, $1 / 3$ of patients were denied the possibility of preserving their anal function.

Although with the LARS score [3] we can now objectively evaluate function after LAR, it remains very difficult to predict anal function before surgery. Male gender, $\mathrm{BMI}>30 \mathrm{~kg} / \mathrm{m}^{2}$ or $\mathrm{ASA} \geq 3$ are well known risk factors for postoperative complications and were also described in this Dutch study. Anastomotic insufficiency is the most feared complication because it is potentially lethal and results in worse anal function or even loss of intestinal continuity. However, LAR with a diverting ileostomy reduced abdominal infectious complications in this study from $16.2 \%$ to $10.1 \%$, which was comparable to LHP $(6.5 \%)$. It seems obvious to conclude that a diverting ileostomy should always be performed in these high risk patients.

The individual decision between a LHP or LAR with ileostomy in low rectal cancer after neoadjuvant therapy remains difficult. Ileostomy closure is not without morbidity [4], 5-10\% of ileostomies never get closed [1] and $20-25 \%$ of patients with a very low anastomosis will require ultimately a stoma $[2,5]$. This must be balanced against up to $50 \%$ of stoma related complications such as parastomal hernia or prolapse requiring re-interventions in approximately $30 \%[5,6]$. Patient preference is a frequently stated reason for one or the other, but the decision is influenced by the surgeons opinion and is very subjective. No patient will have experienced both a colostomy and low anastomosis before rectal cancer surgery and therefore cannot really compare the alternatives. Patients with rectal cancer want to be cured and survive without disease and with a good quality of life for as long as possible. Functional outcomes may improve over time [3]. LHP is certainly an option, but a low anastomosis has in many patients a good long-term outcome providing surgeons minimize postoperative morbidity and mortality.

\section{Dieter Hahnloser}

Department of Visceral Surgery, University Hospital Lausanne, Lausanne, Switzerland

E-mail: dieter.hahnloser@chuv.ch

\section{References}

I Jonker FHW, Tanis PJ, Coene PPLO, Gietelink L, van der Harst E and Dutch Surgical Colorectal Audit Group. Comparison of a low Hartmann's procedure with low colorectal anastomosis with and without defunctioning ileostomy after radiotherapy for rectal cancer: results from a national registry. Colorectal Dis 2016; 18: 785-94.

2 Celerier B, Denost Q, Van Geluwe B, Pontallier A, Rullier E. The risk of definitive stoma formation at 10 years after low and ultralow anterior resection for rectal cancer. Colorectal Dis 2016; 18: 59-66. 
3 Bregendahl S, Emmertsen KJ, Lous J, Laurberg S. Bowel dysfunction after low anterior resection with and without neoadjuvant therapy for rectal cancer: a population-based cross-sectional study. Colorectal Dis 2013; 15: 1130-9.

4 Loffler T, Rossion I, Gooßen K et al. Hand suture versus stapler for closure of loop ileostomy-a systematic review and meta-analysis of randomized controlled trials. Langenbecks Arch Surg 2015; 400: 193-205.
5 Hassan I, Larson DW, Wolff BG et al. Impact of pelvic radiotherapy on morbidity and durability of sphincter preservation after coloanal anastomosis for rectal cancers. Dis Colon Rectum 2008; 51: 32-7.

6 Carne PW, Robertson GM, Frizelle FA. Parastomal hernia. Br J Surg 2003; 90: 784-93. 